\title{
An Evaluation of Scale and Noise Sensitivity of Fibre Orientation Estimation in Volume Images
}

\author{
Maria Axelsson \\ Centre for Image Analysis, Swedish University of Agricultural Sciences, \\ Box 337, SE-751 05, Uppsala, Sweden \\ maria@cb.uu.se
}

\begin{abstract}
Fibre orientation influences many important properties of fibre-based materials, for example, strength and stiffness. Fibre orientation and the orientation anisotropy in paper and other wood fibre-based materials have previously been estimated using two-dimensional images. Recently, we presented a method for estimating the three-dimensional fibre orientation in volume images based on local orientation estimates. Here, we present an evaluation of the method with respect to scale and noise sensitivity. The evaluation is performed for both tubular and solid fibres. We also present a new method for automatic scale selection for solid fibres. The method is based on a segmentation of the fibres that also provides an estimate of the fibre dimension distribution in an image. The results show that the fibre orientation estimation performs well both in noisy images and at different scales. The presented results can be used as a guide to select appropriate parameters for the method when it is applied to real data. The applicability of the fibre orientation estimation to fibre-based materials with solid fibres is demonstrated for a volume image of a press felt acquired with X-ray microtomography.
\end{abstract}

\section{Introduction}

Fibre orientation is an important property of many fibre-based materials. In paper and wood fibre composite materials it influences other physical properties such as strength, stiffness, and hygroexpansion. If the microscopical material properties can be correlated to macroscopical material properties, new materials with more specialised properties can be designed.

Previously, methods for estimating fibre orientation in both two-dimensional (2D) images and three-dimensional (3D) images have been presented. In [1] a sheet splitting method including a 2D gradient image analysis method of the scanned sheets was presented. This is the predominant method used in the paper industry to estimate fibre orientation. Today, fibre orientation estimates can be obtained for A4 or A3 sheet sizes with a spatial resolution of $2 \times 2 \mathrm{~mm}$. For a review see for example [2]. However, only orientations in the plane of the sheet can be estimated, since the $3 \mathrm{D}$ information is lost in the splitting.

Material properties can be studied in 3D using volume images. In 2001 Samuelsen et al. 3] demonstrated that synchrotron X-ray microtomography provides a tool to acquire volume images of materials such as paper. The technique 
is the same as for Computed Tomography (CT) used in medical applications, but the resolution is in the micrometer range, which makes X-ray microtomography suitable to image material microstructure. Today, desktop X-ray microtomography scanners are also available. However, they provide lower resolution compared to synchrotron X-ray microtomography.

2D methods, such as stereological and cross sectional methods, have been proposed for estimating fibre orientation in volume images. However, such methods are not suited to estimate fibre orientation in wood fibre based materials such as paper and wood fibre composites, due to the large shape and size variation of wood fibres after they have passed the pulping process.

Few image analysis methods have been proposed for estimating 3D fibre orientation. A method for synthetic fibres was presented in 4. A fixed number of filters with different orientations were used and the orientation corresponding to the filter with the largest filter response was selected as the fibre orientation in each voxel. This approach is computationally heavy when many filters are used to obtain good angular resolution. In [5] we presented a method for estimating the $3 \mathrm{D}$ fibre orientation in volume images. The method is based on local orientation estimates using quadrature filters and structure tensors. This method provides detailed estimates of the fibre orientation using only six quadrature filters that can estimate any orientation in $3 \mathrm{D}$. Here, we evaluate the robustness of the method with respect to noise and scale using synthetic data. The error estimates for both tubular fibres, such as wood fibres, and for solid fibres, such as synthetic polymer fibres, is evaluated. In addition, we present a novel method for automatic scale selection for solid fibres. We also demonstrate the applicability of the $3 \mathrm{D}$ fibre orientation estimation to press felts, which is a material made of solid polymer fibres.

The method for estimating 3D fibre orientation is outlined in Section 2 and the new method for automatic scale selection for solid fibres is presented in 3 . The evaluation of the orientation estimation with experiments and results is presented in Section 4 and a discussion and conclusion are found in Section 5.

\section{3D Fibre Orientation Estimation}

The fibre orientation in a material can be estimated using the local orientation of the material microstructure in volume images. The method presented in [5] is based on a framework for local orientation estimation using quadrature filters and structure tensors, which is thoroughly described in 6].

The local 3D orientation of the structure in a neighbourhood of a voxel can be estimated using a set of at least six quadrature filters, where each filter is sensitive to signal variation in a certain orientation. The filters are phase invariant where the real part of each filter corresponds to a line detector and the complex part corresponds to an edge detector. The centre frequency of the radial function of the filters should be selected to correspond to the size of the interesting image structures. Small filters that correspond to edges in the image provide better estimates than large filters that correspond to large structures such as the fibre walls. 
The volume image is convolved with the quadrature filters and the filter responses are combined to a structure tensor in each voxel. The structure tensor is represented by a $3 \times 3$ symmetric matrix in $3 \mathrm{D}$. There are thus six independent components. To reduce local errors and obtain better estimates the tensor field is smoothed by convolving each component image with a small Gaussian kernel. The standard deviation for the Gaussian distribution is denoted $\sigma_{s}$. The fibre orientation is a large scale property that is assumed to vary slower than the small scale variations and noise that is reduced by the smoothing.

As described in [6] the local orientation and local structure anisotropy in each voxel neighbourhood can be obtained from the eigenvectors and the eigenvalues of the tensor. The eigenvalues are sorted according to size in descending order. The sorted eigenvalues are denoted $\lambda_{1}, \lambda_{2}$, and $\lambda_{3}$ and the corresponding eigenvectors $\mathbf{e}_{\mathbf{1}}, \mathbf{e}_{\mathbf{2}}$, and $\mathbf{e}_{\mathbf{3}}$. The orientation of the largest signal variation in a neighbourhood, $\mathbf{e}_{\mathbf{1}}$, is referred to as the local orientation. Certainty estimates for the estimated local orientation and the other two orientation estimates are given by

$$
c_{1}=\frac{\lambda_{1}-\lambda_{2}}{\lambda_{1}}, \quad c_{2}=\frac{\lambda_{2}-\lambda_{3}}{\lambda_{1}}, \quad c_{3}=\frac{\lambda_{3}}{\lambda_{1}}
$$

The fibre orientation is modelled as the orientation with the least signal variation. The fibre orientation can thus be estimated using the eigenvector $\mathbf{e}_{\mathbf{3}}$. The $c_{2}$ measurement is used as certainty for the fibre orientation. The certainty is large in neighbourhoods where there is large signal variation in two main orientations and small signal variation in the third orientation. When the orientation estimates are weighted by the $c_{2}$ measurement and also by the probability of the voxel to contain a fibre, the estimation error is reduced [5]. The obtained orientation estimates can be averaged over parts of the sample, like layers of a paper sheet, or over the whole sample depending on the desired output.

\section{Automatic Scale Selection for Solid Fibres}

Fibre orientation estimates from different parameter setups can be combined to improve the estimation result for materials with different fibre dimensions. Here, a method for selecting scale by weighting the orientation estimates from volumes with solid fibres is presented. The method is intended for application to raw data volume images from X-ray microtomography and similar images.

A segmentation of the fibres, where each voxel contains the approximate fibre radius, is calculated using the raw volume image. First noise is reduced using bilateral filtering [7, which is an edge preserving filtering method. Then the volume is segmented into fibre and void using hysteresis thresholding to binarise the samples [8]. The distance transform [10] is applied to the fibres where the shortest distance to the background is calculated for all voxels inside the fibres. Then the watershed segmentation algorithm [9] is applied to the distance transformed image. This creates a regionalisation of the fibres where each maximum in the distance transform generates one region. The distance transform is smoothed before the watershed algorithm is applied to reduce over-segmentation due to 
noise and small shape variations of the fibres. A segmented image where each voxel contains the approximate fibre radius is obtained by setting all voxels in a region to the value of the corresponding distance maximum. The distance maxima in the segmented image are used to weight orientation results obtained using different parameter setups for different fibre dimensions. This is exemplified for three fibre diameters in Section 4.2. If the diameters are not known exactly or assumed to vary, results obtained using a range of parameter setups can also be combined using this method. In addition, the presented segmentation of the fibre dimensions directly provides an estimate of the fibre dimension distribution by summing the number of voxels for each fibre radius and weighting the result with the corresponding radius.

\section{Experiments and Results}

The evaluation of the orientation estimation using synthetic data is presented in Section 4.1 and the applicability of the orientation estimation to real data with solid fibres is illustrated in Section 4.2 .

\subsection{Synthetic Data}

Synthetic test volumes with randomly positioned straight fibres were used to evaluate the fibre orientation estimation. Volumes with either tubular or solid fibres were generated with specific fibre dimensions and known fibre orientation. To evaluate the difference between tubular and solid fibres, pairs of volumes with the same fibre positions and fibre orientation but different fibre interior were used. The volumes were $250 \times 250 \times 250$ voxels with approximately 150 500 fibres in each volume, depending on the fibre diameter. The fibre length was constant in all volumes. Six different fibre diameters, $D_{f}$, were used. These correspond to volumes with different fibre dimensions or different scales. A test set with ten volumes for each fibre diameter was generated to provide good statistics. Noise was also added to the volumes in the test set to evaluate the robustness of the method. The original pixel values range from 0 to 1 with the background close to 0 and the fibres close to 1 . Gaussian noise with zero mean and standard deviation, $\sigma_{n}$, of $0.2,0.4$, and 0.6 was added to each volume.

Fibre orientation was estimated in the four test sets; in the images without noise and in the images with the three different noise levels. The spatial filter size was $7 \times 7 \times 7$ voxels, the centre frequency $\pi / 2$, and the bandwidth 2 octaves. The weighted mean error for the estimated fibre orientation is calculated as

$$
E_{s\left(\sigma_{s}\right)}=\frac{\sum_{I} w c_{2} \arccos \left(\left|\mathbf{v}_{\mathbf{g t}} \cdot \mathbf{e}_{\mathbf{3}}\right|\right)}{\sum_{I} w c_{2}},
$$

where $\sigma_{s}$ is the standard deviation used for the Gaussian in the smoothing of the tensor field, $w$ is a weight depending on the image intensity or the probability of the voxel to contain fibres, $c_{2}$ is the certainty value, $\mathbf{v}_{\mathbf{g t}}$ is the ground truth orientation, and $\mathbf{e}_{\mathbf{3}}$ is the estimated orientation. The weighted mean error was 
Table 1. Comparison of the estimation error for tubular fibres of different dimensions. No noise. Average over ten volumes.

\begin{tabular}{|r|r|rrrrrrrrr|}
\hline$D_{f}$ & $E$ & $E_{s(0)}$ & $E_{s(1)}$ & $E_{s(2)}$ & $E_{s(3)}$ & $E_{s(4)}$ & $E_{s(5)}$ & $E_{s(6)}$ & $E_{s(7)}$ & $E_{s(8)}$ \\
\hline \hline 6 & 15.85 & 9.55 & 3.85 & $\mathbf{2 . 3 0}$ & 3.00 & 4.58 & 6.72 & 9.10 & 11.48 & 13.75 \\
8 & 15.68 & 12.99 & 5.26 & $\mathbf{1 . 4 0}$ & 1.89 & 3.03 & 4.65 & 6.56 & 8.59 & 10.63 \\
10 & 12.80 & 14.05 & 8.14 & 2.63 & $\mathbf{1 . 6 4}$ & 2.41 & 3.57 & 5.07 & 6.76 & 8.53 \\
12 & 19.01 & 17.89 & 12.15 & 4.92 & $\mathbf{1 . 7 4}$ & 1.97 & 2.77 & 3.82 & 5.09 & 6.51 \\
14 & 21.18 & 20.16 & 14.47 & 8.20 & 3.29 & $\mathbf{1 . 9 0}$ & 2.39 & 3.20 & 4.20 & 5.36 \\
16 & 21.78 & 23.43 & 18.05 & 11.58 & 5.08 & 2.21 & $\mathbf{2 . 1 9}$ & 2.85 & 3.64 & 4.60 \\
\hline
\end{tabular}

Table 2. Comparison of the estimation error for solid fibres of different dimensions. No noise. Average over ten volumes.

\begin{tabular}{|r|r|rrrrrrrrr|}
\hline \multicolumn{1}{|r}{$D_{f}$} & \multicolumn{1}{|c|}{$E$} & $E_{s(0)}$ & $E_{s(1)}$ & $E_{s(2)}$ & $E_{s(3)}$ & $E_{s(4)}$ & $E_{s(5)}$ & $E_{s(6)}$ & $E_{s(7)}$ & $E_{s(8)}$ \\
\hline \hline 6 & 9.56 & 6.72 & 3.31 & $\mathbf{2 . 1 8}$ & 3.04 & 4.74 & 6.91 & 9.26 & 11.62 & 13.86 \\
8 & 11.94 & 10.28 & 5.94 & 3.03 & $\mathbf{2 . 4 9}$ & 3.49 & 5.05 & 6.90 & 8.88 & 10.88 \\
10 & 13.88 & 14.73 & 9.44 & 5.38 & 3.19 & $\mathbf{3 . 1 3}$ & 4.16 & 5.58 & 7.20 & 8.90 \\
12 & 23.94 & 20.66 & 14.50 & 9.16 & 5.42 & $\mathbf{3 . 7 5}$ & $\mathbf{3 . 7 5}$ & 4.58 & 5.74 & 7.06 \\
14 & 28.77 & 23.94 & 18.00 & 12.53 & 8.03 & 5.09 & $\mathbf{4 . 0 7}$ & 4.27 & 5.07 & 6.09 \\
16 & 31.39 & 26.65 & 21.68 & 16.17 & 11.39 & 7.48 & 5.29 & $\mathbf{4 . 6 4}$ & 4.89 & 5.63 \\
\hline
\end{tabular}

Table 3. Comparison of the estimation error for tubular fibres of different dimensions. Noise with $\sigma_{n}=0.2$ was added to the images. Average over ten volumes.

\begin{tabular}{|r|c|rrrrrrrrr|}
\hline$D_{f}$ & $E$ & $E_{s(0)}$ & $E_{s(1)}$ & $E_{s(2)}$ & $E_{s(3)}$ & $E_{s(4)}$ & $E_{s(5)}$ & $E_{s(6)}$ & $E_{s(7)}$ & $E_{s(8)}$ \\
\hline \hline 6 & 24.83 & 19.10 & 6.17 & $\mathbf{3 . 6 0}$ & 4.02 & 5.45 & 7.53 & 9.90 & 12.33 & 14.65 \\
8 & 29.24 & 25.08 & 8.31 & $\mathbf{2 . 8 2}$ & $\mathbf{2 . 8 2}$ & 3.72 & 5.20 & 7.07 & 9.10 & 11.16 \\
10 & 32.14 & 28.65 & 12.30 & 4.07 & $\mathbf{2 . 7 6}$ & 3.22 & 4.18 & 5.55 & 7.18 & 8.93 \\
12 & 35.68 & 33.59 & 18.48 & 6.92 & 3.22 & $\mathbf{3 . 1 2}$ & 3.67 & 4.56 & 5.73 & 7.09 \\
14 & 39.29 & 38.25 & 22.58 & 11.01 & 4.94 & $\mathbf{3 . 3 3}$ & 3.50 & 4.09 & 4.93 & 5.98 \\
16 & 41.05 & 41.07 & 27.63 & 15.53 & 7.13 & 3.90 & $\mathbf{3 . 5 4}$ & 3.92 & 4.56 & 5.39 \\
\hline
\end{tabular}

Table 4. Comparison of the estimation error for solid fibres of different dimensions. Noise with $\sigma_{n}=0.2$ was added to the images. Average over ten volumes.

\begin{tabular}{|r|c|rrrrrrrrr|}
\hline$D_{f}$ & $E$ & $E_{s(0)}$ & $E_{s(1)}$ & $E_{s(2)}$ & $E_{s(3)}$ & $E_{s(4)}$ & $E_{s(5)}$ & $E_{s(6)}$ & $E_{s(7)}$ & $E_{s(8)}$ \\
\hline \hline 6 & 25.41 & 21.06 & 6.81 & $\mathbf{3 . 6 5}$ & 3.96 & 5.43 & 7.51 & 9.85 & 12.21 & 14.47 \\
8 & 34.13 & 31.85 & 12.44 & 5.43 & $\mathbf{4 . 0 5}$ & 4.62 & 5.96 & 7.71 & 9.65 & 11.63 \\
10 & 40.26 & 40.11 & 21.29 & 8.98 & 5.04 & $\mathbf{4 . 4 6}$ & 5.17 & 6.40 & 7.91 & 9.55 \\
12 & 44.94 & 45.43 & 31.23 & 15.13 & 7.95 & 5.39 & $\mathbf{5 . 0 7}$ & 5.70 & 6.72 & 7.95 \\
14 & 47.16 & 47.89 & 36.68 & 21.26 & 11.46 & 6.92 & 5.43 & $\mathbf{5 . 4 1}$ & 6.03 & 6.93 \\
16 & 48.64 & 49.67 & 41.17 & 28.43 & 16.79 & 10.06 & 6.96 & $\mathbf{6 . 0 0}$ & 6.10 & 6.70 \\
\hline
\end{tabular}


Table 5. Comparison of the estimation error for tubular fibres of different dimensions. Noise with $\sigma_{n}=0.4$ was added to the images. Average over ten volumes.

\begin{tabular}{|r|c|rrrrrrrrr|}
\hline$D_{f}$ & $E$ & $E_{s(0)}$ & $E_{s(1)}$ & $E_{s(2)}$ & $E_{s(3)}$ & $E_{s(4)}$ & $E_{s(5)}$ & $E_{s(6)}$ & $E_{s(7)}$ & $E_{s(8)}$ \\
\hline \hline 6 & 36.21 & 33.09 & 11.63 & 6.16 & $\mathbf{5 . 8 8}$ & 6.94 & 8.77 & 10.98 & 13.30 & 15.55 \\
8 & 39.50 & 37.52 & 14.60 & 5.27 & $\mathbf{4 . 4 5}$ & 4.99 & 6.22 & 7.90 & 9.81 & 11.79 \\
10 & 42.01 & 40.64 & 20.05 & 6.92 & $\mathbf{4 . 6 4}$ & $\mathbf{4 . 6 4}$ & 5.31 & 6.45 & 7.91 & 9.54 \\
12 & 44.37 & 43.75 & 27.37 & 10.48 & 5.47 & $\mathbf{4 . 7 7}$ & 5.03 & 5.72 & 6.71 & 7.93 \\
14 & 47.21 & 47.01 & 32.41 & 15.48 & 7.57 & 5.31 & $\mathbf{5 . 0 3}$ & 5.37 & 6.02 & 6.91 \\
16 & 48.31 & 48.33 & 36.55 & 20.72 & 10.09 & 6.13 & $\mathbf{5 . 2 4}$ & 5.32 & 5.76 & 6.45 \\
\hline
\end{tabular}

Table 6. Comparison of the estimation error for solid fibres of different dimensions. Noise with $\sigma_{n}=0.4$ was added to the images. Average over ten volumes.

\begin{tabular}{|r|c|rrrrrrrrr|}
\hline$D_{f}$ & $E$ & $E_{s(0)}$ & $E_{s(1)}$ & $E_{s(2)}$ & $E_{s(3)}$ & $E_{s(4)}$ & $E_{s(5)}$ & $E_{s(6)}$ & $E_{s(7)}$ & $E_{s(8)}$ \\
\hline \hline 6 & 39.57 & 37.34 & 14.38 & 7.04 & $\mathbf{6 . 2 2}$ & 7.12 & 8.82 & 10.91 & 13.10 & 15.23 \\
8 & 45.12 & 44.16 & 24.12 & 9.98 & 6.86 & $\mathbf{6 . 6 7}$ & 7.55 & 9.00 & 10.73 & 12.57 \\
10 & 48.39 & 48.13 & 33.77 & 14.99 & 8.34 & $\mathbf{6 . 8 0}$ & 6.93 & 7.78 & 9.03 & 10.48 \\
12 & 50.58 & 50.59 & 40.56 & 22.93 & 11.97 & 8.16 & $\mathbf{7 . 2 2}$ & 7.43 & 8.18 & 9.23 \\
14 & 51.68 & 51.79 & 43.93 & 29.73 & 16.22 & 9.98 & 7.76 & $\mathbf{7 . 2 7}$ & 7.55 & 8.22 \\
16 & 52.60 & 52.75 & 46.74 & 36.52 & 22.66 & 13.76 & 9.70 & 8.23 & $\mathbf{7 . 9 8}$ & 8.29 \\
\hline
\end{tabular}

Table 7. Comparison of the estimation error for tubular fibres of different dimensions. Noise with $\sigma_{n}=0.6$ was added to the images. Average over ten volumes.

\begin{tabular}{|r|c|rrrrrrrrr|}
\hline$D_{f}$ & $E$ & $E_{s(0)}$ & $E_{s(1)}$ & $E_{s(2)}$ & $E_{s(3)}$ & $E_{s(4)}$ & $E_{s(5)}$ & $E_{s(6)}$ & $E_{s(7)}$ & $E_{s(8)}$ \\
\hline \hline 6 & 44.01 & 42.69 & 20.22 & 9.92 & $\mathbf{8 . 4 5}$ & 8.99 & 10.44 & 12.36 & 14.45 & 16.55 \\
8 & 45.27 & 44.62 & 22.79 & 8.45 & $\mathbf{6 . 4 1}$ & 6.51 & 7.46 & 8.93 & 10.69 & 12.55 \\
10 & 47.42 & 47.00 & 28.87 & 11.00 & 6.93 & $\mathbf{6 . 3 0}$ & 6.64 & 7.54 & 8.80 & 10.27 \\
12 & 49.04 & 48.92 & 35.39 & 15.86 & 8.34 & 6.65 & $\mathbf{6 . 4 9}$ & 6.92 & 7.73 & 8.81 \\
14 & 51.14 & 51.12 & 39.71 & 22.08 & 11.38 & 7.85 & 6.91 & $\mathbf{6 . 8 8}$ & 7.29 & 8.00 \\
16 & 51.77 & 51.83 & 42.33 & 27.48 & 14.55 & 9.07 & 7.32 & $\mathbf{6 . 9 2}$ & 7.09 & 7.58 \\
\hline
\end{tabular}

Table 8. Comparison of the estimation error for solid fibres of different dimensions. Noise with $\sigma_{n}=0.6$ was added to the images. Average over ten volumes.

\begin{tabular}{|r|c|rrrrrrrrr|}
\hline$D_{f}$ & $E$ & $E_{s(0)}$ & $E_{s(1)}$ & $E_{s(2)}$ & $E_{s(3)}$ & $E_{s(4)}$ & $E_{s(5)}$ & $E_{s(6)}$ & $E_{s(7)}$ & $E_{s(8)}$ \\
\hline \hline 6 & 46.92 & 46.02 & 25.17 & 12.32 & $\mathbf{9 . 7 7}$ & 9.86 & 11.01 & 12.67 & 14.55 & 16.45 \\
8 & 50.13 & 49.75 & 35.07 & 17.05 & 11.16 & $\mathbf{9 . 8 1}$ & 10.03 & 11.02 & 12.42 & 14.01 \\
10 & 51.91 & 51.78 & 41.68 & 23.65 & 13.51 & 10.40 & $\mathbf{9 . 6 6}$ & 9.95 & 10.81 & 11.97 \\
12 & 53.16 & 53.15 & 45.85 & 31.75 & 18.16 & 12.39 & 10.38 & $\mathbf{9 . 9 0}$ & 10.19 & 10.92 \\
14 & 53.77 & 53.80 & 47.81 & 36.98 & 22.92 & 14.69 & 11.29 & 10.05 & $\mathbf{9 . 8 2}$ & 10.13 \\
16 & 54.38 & 54.42 & 49.67 & 41.90 & 29.57 & 19.10 & 13.79 & 11.48 & 10.63 & $\mathbf{1 0 . 5 3}$ \\
\hline
\end{tabular}




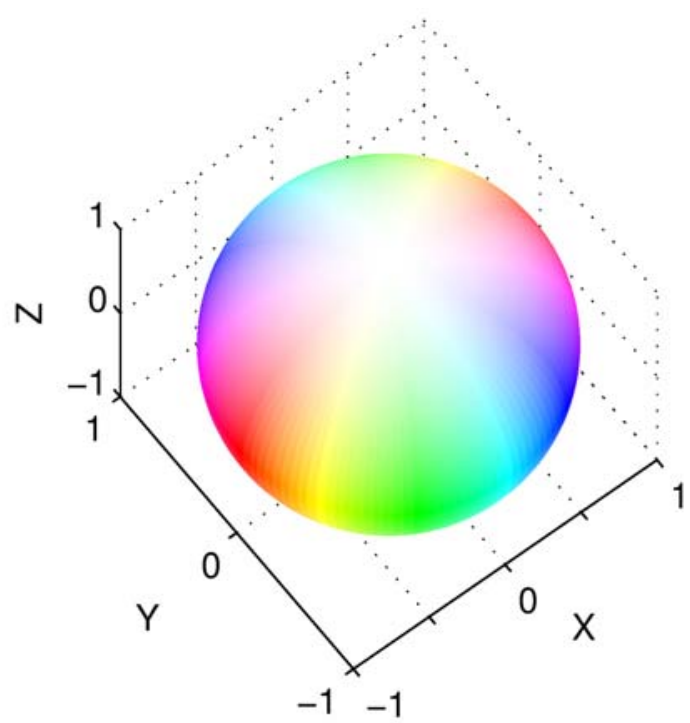

Fig. 1. Colour map for the estimated orientations. Hue varies with the $X$ and $Y$ coordinates and saturation with the $Z$ coordinate.

calculated for each volume image both before smoothing the tensor field, $E_{s(0)}$, and after eight different amounts of Gaussian smoothing, $E_{s\left(\sigma_{s}\right)}$, with standard deviation $\sigma_{s}$. The mean error without weighting the estimates, $E$, was also calculated. The results for the noise free volumes are reported in Table 1 and Table 2 for the tubular and solid fibres, respectively. All errors are reported in degrees. The minimal error is indicated using bold face. The results for the three noise levels for both fibre types are reported in Table 3 to Table 8 .

Smoothing the tensor field prior to estimating the orientation reduces the error for all fibre diameters, $D_{f}$, and all noise levels. Larger smoothing must be used for larger values of $D_{f}$ to obtain the lower errors. The error increases with larger amounts of noise as expected, but the method still performs well for the largest amount of noise. The error is larger for solid fibres than for tubular fibres for larger fibre diameters. For smaller diameters, such as $D_{f}=6$ voxels, the difference between solid fibres and tubular fibres is small. It is evident from the results that high resolution of the fibre cross section does not contribute to better estimates. As a result, high resolution images can be downsampled while maintaining the performance of the method.

\subsection{Real Data}

Press felts that are used in paper manufacturing are made of synthetic polymer fibres with different fibre diameters. They consist of both larger fibres, that form the base weave, and fibres with smaller diameters, called batt fibres, that are needled onto the base weave. A volume image of a press felt was acquired using desktop X-ray microtomography in a Skyscan 1172. Fibres with four different 


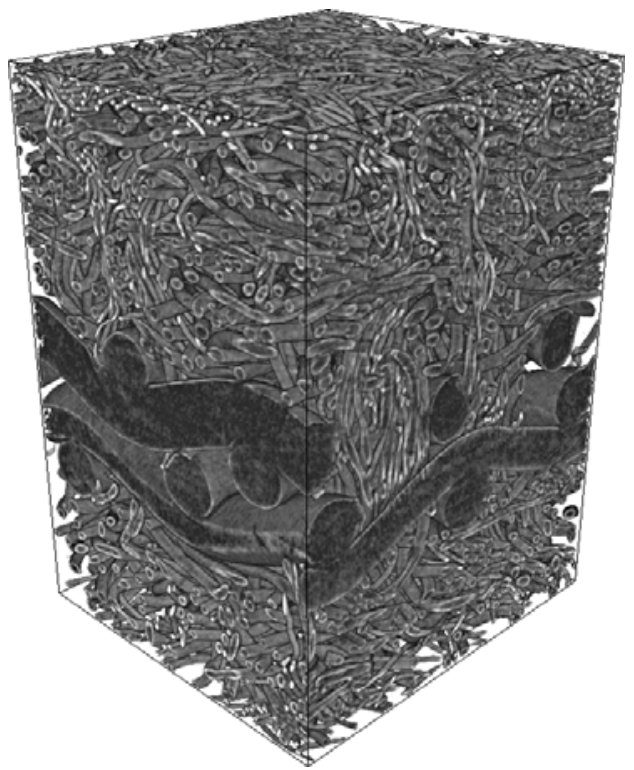

(a)

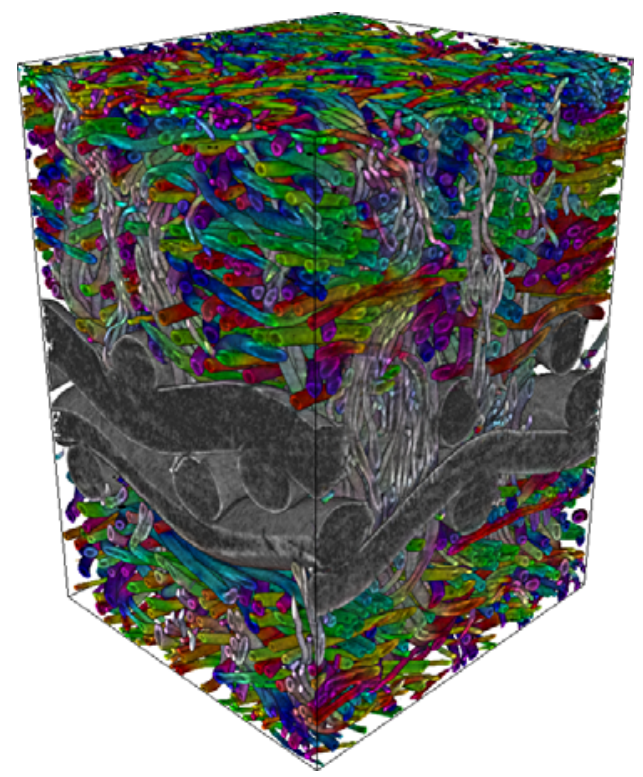

(b)

Fig. 2. (a) Volume rendering of a press felt, which consist of polymer fibres with four different fibre diameters. (b) Volume rendering of the $3 \mathrm{D}$ fibre orientation estimates in the same volume.

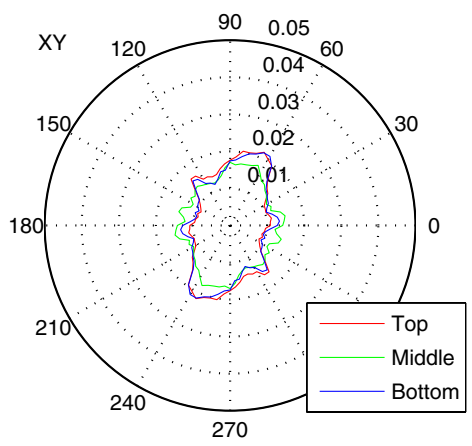

(a)

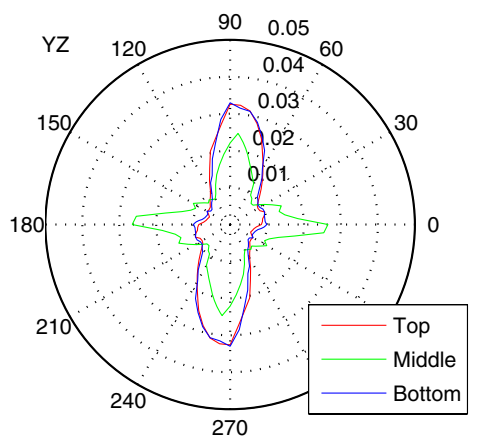

(b)

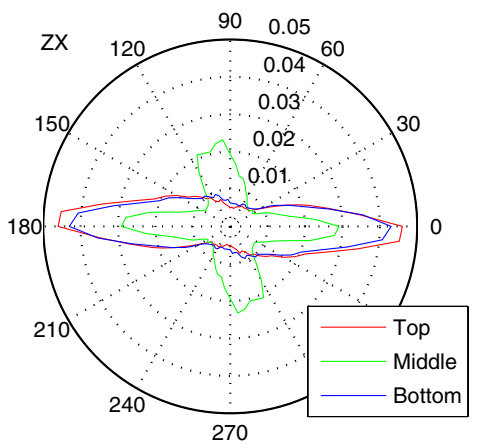

(c)

Fig. 3. The fibre orientation distribution for the top, middle, and bottom part of the sample. (a) Projection on the $X Y$ plane. (b) Projection on the $Y Z$ plane. (c) Projection on the $Z X$ plane. 
sizes were present in the image; the fibres in the base weave and batt fibres of three different diameters. As the results have shown that high resolution of individual fibre cross sections is not needed to estimate fibre orientation, the volume image was downsampled to match the fibre diameters in the experiments on synthetic data. The resulting image size was $467 \times 501 \times 642$ voxels, with batt fibre diameters of 6,12 , and 15 voxels. Figure2(a) shows a volume rendering of the material. The difference in fibre diameter between the base weave and the batt fibres is clearly visible in the figure. The orientation of the fibres in the base weave are not estimated in this experiment, since they are large compared to the batt fibres and only partly visible in the image. Larger samples acquired at lower resolution are needed to obtain good estimates of the fibre orientation of the base weave fibres.

Fibre orientation was estimated in the volume image using the same parameter setup for the filters as in Section 4.1. Three values of $\sigma_{s}, 2,4$, and, 6 voxels, corresponding to the three batt fibre diameters were selected using the three lowest error estimates from Table 2, The resulting tensor images were combined to a final result by selecting orientation estimates voxel-wise from each of the three images using the smallest difference between the optimal radius for each of the three scales and the fibre radius obtained using the method presented in Section 3. The fibres in the base weave were also masked using the estimated fibre radii. The final result of the estimated orientation is shown in Figure 2(b). The base weave is shown in gray and the batt fibres are pseudo coloured based on the estimated orientation as proposed in [5]. Hue varies with the $X$ and $Y$ coordinates and saturation with the $Z$ coordinate, see Figure 1 Figure 3 shows the distribution of the estimated orientations projected on the $X Y, Y Z$, and $Z X$ planes for the top, middle, and bottom part of the sample. More fibres are orientated in the $X$ direction in all parts. In the middle part, the fibre orientations are limited by the batt fibres and the fibres are oriented both in the $X$ direction and the $Z$ direction.

\section{Discussion and Conclusion}

In this paper we have shown that the method for 3D fibre orientation estimation [5] is applicable to both tubular and solid fibres. The method was evaluated in a study on synthetic data with respect to noise and scale. Ten repetitions of each parameter setup was used to obtain reliable results. The results show that the method provides good orientation estimates for both tubular and solid fibres in both noise free and noisy images. It also performs well for a large range of fibre diameters which correspond to different scales. As the fibre orientation provides good estimates also for the smaller fibre diameters it is possible to use low resolution volumes of the fibre material to estimate fibre orientation. The model of the fibre orientation that is used in the method is valid also for small fibre cross sections. The results presented in this paper can be used as a guide to select appropriate parameters for real data. In addition, we have demonstrated the suitability of the method to materials based on solid fibres using press felt. 
It was exemplified how parameter selection can be performed using real data. A new method for estimating the fibre radii for solid fibres, which can be used to select scale locally in the image, was also presented and exemplified.

It is left for future work to develop methods for automatic parameter selection, where suitable parameters are selected directly using image information. Another interesting topic is more advanced adaptive scale selection which is based on the local fibre dimensions. This is useful for materials that consist of fibres with different fibre dimensions.

Acknowledgements. The author acknowledges Prof. Gunilla Borgefors and Dr. Joakim Rydell for valuable scientific discussions. The press felt volume image was provided by Albany International AB in Halmstad, Sweden.

\section{References}

1. Erkkilä, A.-L., Pakarinen, P., Odell, M.: Sheet forming studies using layered orientation analysis. Pulp and Paper Canada 99(1), 81-85 (1998)

2. Hirn, U., Bauer, W.: Evaluating an improved method to determine layered fibre orientation by sheet splitting. In: 61st Appita Annual Conference and Exhibition, Gold Coast, Australia, May 6-9, pp. 71-79 (2007)

3. Samuelsen, E., Gregersen, Ø., Houen, P.J., Helle, T., Raven, C., Snigirev, A.: Threedimensional imaging of paper by use of synchroton X-Ray microtomography. Journal of Pulp and Paper Science 27(2), 50-53 (2001)

4. Robb, K., Wirjandi, O., Schladitz, K.: Fiber orientation estimation from 3D image data: Practical algorithms, visualization, and interpretation. In: Proceedings of 7th International Conference on Hybrid Intelligent Systems, September 2007, pp. 320-325 (2007)

5. Axelsson, M.: Estimating 3D Fibre Orientation in Volume Images. In: Proceedings of 19th International Conference on Pattern Recognition (2008)

6. Granlund, G.H., Knutsson, H.: Signal Processing for Computer Vision. Kluwer Academic Publishers, Dordrecht (1995)

7. Tomasi, C., Manduchi, R.: Bilateral filtering for gray and color images. In: IEEE International Conference on Computer Vision 1998, pp. 836-846 (1998)

8. Canny, J.F.: A computational approach to edge detection. IEEE Transactions on Pattern Analysis and Machine Intelligence 8(6), 679-698 (1986)

9. Vincent, L., Soille, P.: Watersheds in digital spaces: An efficient algorithm based on immersion simulations. IEEE Transactions on Pattern Analysis and Machine Intelligence 13(6), 583-598 (1991)

10. Borgefors, G.: On Digital Distance Transforms in Three Dimensions. Computer Vision and Image Understanding 64(3), 368-376 (1996) 